Research in Astronomy and Astrophysics manuscript no.

(LTEX: main.tex; printed on February 20, 2019; 1:47)

\title{
The r-mode instability windows of strange stars
}

\author{
Yu-Bin Wang ${ }^{1,2}$, Xia Zhou $^{2,3}$, Na Wang ${ }^{2,3}$ and Xiong-Wei Liu ${ }^{1}$ \\ ${ }^{1}$ Physics and Space Science College, China West Normal University, Nanchong 637002, China; \\ 2 Xinjiang Astronomical Observatory, Chinese Academy of Sciences, Urumqi 830011, China \\ zhouxia@xao.ac.cn \\ 3 Key Laboratory of Radio Astronomy, Chinese Academy of Sciences, Beijing 100049, China
}

Received 2018 August 14; accepted 2018 October 10

\begin{abstract}
With the constraint from gravitational wave emission of a binary merger system (GW170817) and two-solar-mass pulsar observations, we investigate the r-mode instability windows of strange stars with unpaired and color-flavor-locked phase strange quark matter. Shear viscosities due to surface rubbing and electron-electron scattering are taken into account in this work. The results show that the effects of the equation of state of unpaired strange quark matter are only dominant at low temperature, but do not have significant effects on strange stars in the color-flavor-locked phase. A color-flavor-locked phase strange star, which is surrounded by an insulating nuclear crust, seems to be consistent with observational data of young pulsars. We find that an additional enhanced dissipation mechanisms might exist in SAX J1808.4-3658. Fast spinning young pulsar PSR J0537-6910 is a primary source for detecting gravitational waves from a rotating strange star, and young pulsars might be strange stars with color-flavor-locked phase strange quark matter.
\end{abstract}

Key words: stars: neutron — equation of state — stars: oscillations

\section{INTRODUCTION}

The r-modes are non-radial pulsations of compact stars that are primarily driven by Coriolis forces and are coupled to gravitational radiation (Chandrasekhar 1970; Friedman \& Schutz 1978; Andersson 1998; Friedman \& Morsink 1998; Owen et al. 1998; Andersson et al 2002; Alford \& Schwenzer 2014a). The $r$-mode instability only happens in a range of spin frequencies and temperatures, which is determined by a competition between the effects of gravitational radiation and viscous dissipation damping on modes (Lindblom et al. 1998). Therefore, $r$-mode instability is an important primary physical mechanism that can prevent pulsars from spinning up to their Kepler frequency, which also affects the spinning evolutions of pulsars (Madsen 1998; Andersson et al. 1999; Wang \& Dai 2017). Meanwhile, gravitational waves emitted during the instability process could be detected (Owen et al. 1998; Andersson et al 2002; Mahmoodifar \& 
Such r-modes are damped by viscous dissipation mechanisms (Lindblom et al. 1998), and therefore are connected with microscopic properties of matter inside the stars, which depend on the low energy degrees of freedom and the equation of state (EOS), thus affecting the macroscopic and observable properties of the star. Many studies have tried to constrain the physics behind r-mode instability in compact stars, especially the EOS of cold dense matter, by comparing the r-mode instability windows with the spin frequency and surface temperature of these systems (Becker 2009; Haskell et al. 2012; Mahmoodifar \& Strohmayer 2013; Gusakov et al. 2014; Pi et al. 2015; Kantor et al. 2016; Chugunov et al. 2017).

The LIGO/Virgo detection of gravitational waves from a binary merger system, GW170817, has put a clean and strong constraint on the tidal deformability of the merging objects (Abbott et al. 2017). Combined with two-solar-mass pulsar observations and tidal deformability constraints, Zhou et al. (2018) constrains the parameters of the quark star EOS. In this paper, we will investigate the r-mode instability window of strange stars with unpaired and color-flavor-locked (CFL) phase strange quark matter (SQM). We will utilize a realistic EOS and the new limit on EOS parameters to discuss its effect on the r-mode instability windows of strange star. In the following, we adopt the abbreviations USS for strange star with unpaired SQM, and CSS for strange star with CFL phase SQM.

Most of the uncertainty related to r-mode instability has to do with its damping mechanisms. In a minimum physics model that assumes dissipation only due to standard shear and bulk viscosity, the r-mode instability should be operating in a large portion of the low mass X-ray binary (LMXB) population and in some young sources (Kokkotas \& Schwenzer 2016). Some works showed that the minimum frequency of the instability region of USS $\left(\nu_{\min } \approx 200 \mathrm{~Hz}\right)$ is higher than the rotational frequencies of all known young pulsars, and USS can be consistent with both the observed radio and X-ray data (Alford \& Schwenzer 2014b), except for some special sources. CSS without a crust would have a critical frequency at which the r-mode instability sets in measured in $\mathrm{Hz}$ or fractions of $\mathrm{Hz}$, in gross disagreement with the data on spin frequencies of both X-ray and radio pulsars (Alford et al. 2008). Strange stars can support a thin nuclear crust (Glendenning \& Weber 1992), which only leads to minor changes in the maximum mass compared with bare strange stars (Zdunik 2002). The crust on a USS does not have significant effects on the dissipation of r-modes (Andersson et al 2002). As other sources of viscosity are exponentially suppressed in the case of the CFL phase, some viscosity results from the crust might become dominant in the case of the CFL phase, such as shear due to electron-electron scattering or by surface rubbing at low temperature (Madsen 2000). These viscosities may have significant effects on the r-mode instability windows of a CSS, leading its behavior to agree with observational data of young radio pulsars. Thus, we will investigate the effect of shear viscosity due to electron-electron scattering or by surface rubbing, by comparing the r-mode instability windows of CSS with observational data of young pulsars, and the USS without a crust will be reviewed by comparing with the observational data of LMXBs.

The paper is organized as follows: In Section 2, we briefly review the EOS of unpaired and CFL phase SQM, and give reasonable parameters which are constrained by observations. The main results are presented in Section 3, where r-mode instability windows of USS and CSS are provided with different parameters for the EOS. All of the windows are confronted with the tidal deformability measurement from GW170817 and 
the theoretical r-mode instability windows with the spin frequency and temperature of compact stars in LMXBs and young pulsars. Finally, conclusions and discussion are given in Sec.4

\section{EOS MODELS OF STRANGE STARS}

We use the simple but widely used MIT model to describe the unpaired SQM as a mixture of quarks $(\mathrm{u}, \mathrm{d}, \mathrm{s})$ and electrons (e). The grand canonical potential per unit volume can be written as (Weissenborn et al. 2011; Bhattacharyya et al. 2016)

$$
\Omega_{\mathrm{QM}}=\sum_{i=u, d, s, e} \Omega_{i}^{0}+\frac{3 \mu^{4}}{4 \pi^{2}}\left(1-a_{4}\right)+B_{\mathrm{eff}}
$$

where $\Omega_{i}^{0}$ is the grand canonical potential of quarks (u,d,s) and electrons (e) which are regarded as an ideal relativistic Fermi gas. The second term is characterized by the perturbative quantum chromodynamics (QCD) corrections of gluon mediated quark interactions to $O\left(\alpha_{s}^{2}\right)$ (Fraga et al. 2001; Bhattacharyya et al. 2016) and the parameter $a_{4}=1-2 \alpha_{\mathrm{s}} / \pi$ represents the degree of quark interaction correction in perturbative QCD (Alford et al. 2008). $\mu=\left(\mu_{\mathrm{u}}+\mu_{\mathrm{d}}+\mu_{\mathrm{s}}\right) / 3.0$ is the baryon chemical potential with the total baryon number density $n_{\mathrm{B}}=\left(n_{\mathrm{u}}+n_{\mathrm{d}}+n_{\mathrm{s}}\right) / 3 . B_{\text {eff }}$ is the effective bag constant which includes non-perturbative QCD effects in a phenomenological way.

In the calculation we take $\mathrm{m}_{\mathrm{u}}=\mathrm{m}_{\mathrm{d}}=0$ and $\mathrm{m}_{\mathrm{e}}=0$ (Weissenborn et al. 2011; Bhattacharyya et al. 2016; Li et al. 2017; Zhou et al. 2018). The current mass of strange quark $m_{\mathrm{s}}$ has been constrained well with a recent result of $96_{-4}^{+8} \mathrm{MeV}$ (Aaij et al. 2016). The effect of introducing a finite strange quark mass has been discussed in Zhou et al. (2018) and the results show that a larger $m_{\mathrm{s}}$ will soften the EOS and $\Lambda(1.4)$ only weakly depends on $m_{\mathrm{s}}$. In the following, we set $m_{\mathrm{s}}$ as $100 \mathrm{MeV}$.

At large densities, such that up, down and strange quarks bind together, the condensation term, contained in $3 \Delta^{2} \mu^{2} / \pi^{2}$, emerges to decrease the free energy of quarks, which are assumed to undergo pairing and form the so-called CFL phase (Rajagopal \& Wilczek 2001). As a result, $\Omega_{\mathrm{CFL}}$ can be obtained from (Alford et al. 2001; Weissenborn et al. 2011)

$$
\Omega_{\mathrm{CFL}}=\Omega_{\mathrm{QM}}-\frac{3}{\pi^{2}} \Delta^{2} \mu^{2},
$$

where $\Delta$ is the pairing energy gap for the CFL phase, which lacks an accurate calculation within a typical range $(0-150 \mathrm{MeV})$.

Different choices of parameters for the effective bag constant ( $\left.B_{\text {eff }}\right)$, the perturbative QCD correction parameter $\left(a_{4}\right)$ and the pairing energy gap $(\Delta)$ will lead to various EOS models. These parameters are all confronted with the tidal deformability $(\Lambda)$ measurement from GW170817 and other pulsar observations for systematic constraints on those parameters. As discussed in Zhou et al. (2018), normal quark matter model

parameters are compatible with $B_{\text {eff }}^{1 / 4} \in(134.1,141.4) \mathrm{MeV}$ and $a_{4} \in(0.56,0.91)$ which are derived by considering the GW170817 constraint on $\Lambda(1.4)^{1}$ and two-solar-mass constraint on $M_{\text {TOV }}$ as well as the stability window for quark matter("two flavor line" and "three flavor line"). The possible constraint on the pairing energy gap $\Delta$ might be larger than $50 \mathrm{MeV}$ in the case of $a_{4}=1$, and no new lower limit is found for the gap parameter $\Delta$ in the case of $a_{4}=0.61$ (Zhou et al. 2018). 


\section{THE R-MODE INSTABILITY WINDOWS OF STRANGE STARS}

The r-mode instability window is defined as

$$
\frac{1}{\tau_{\mathrm{gw}}}+\frac{1}{\tau_{\mathrm{V}}}=0
$$

and yields a curve that depends on spin frequency and core temperature. The resulting instability parameter space is commonly depicted as a "window" in the $\nu-T$ plane. Above this curve, the instability condition is satisfied and the star can emit gravitational waves. $\tau_{\mathrm{gw}}$ is the growth time due to emission of gravitational waves (Lindblom et al. 1998; Owen et al. 1998)

$$
\frac{1}{\tau_{\mathrm{gw}}}=-\frac{32 \pi G \Omega^{2 l+2}}{c^{2 l+3}} \frac{(l-1)^{2 l}}{[(2 l+1) ! !]^{2}}\left(\frac{l+2}{l+1}\right)^{2 l+2} \int_{0}^{R} \rho r^{2 l+2} d r,
$$

where $G$ is the gravitational constant and $\rho$ is the stellar density. The viscous damping timescale $\tau_{\mathrm{V}}$ is given by

$$
\frac{1}{\tau_{\mathrm{V}}}=\sum_{\mathrm{i}} \frac{1}{\tau_{\mathrm{i}}}
$$

where the summation is over various dissipation channels(denoted with " $i$ "). In the most common case, in which several dissipation mechanisms are operating simultaneously the viscous timescales are combined according to the parallel resistors rule. For the simplest strange star models, two kinds of viscosity are normally considered: bulk viscosity $\tau_{\mathrm{bv}}$ and shear viscosity $\tau_{\mathrm{sv}}$.

Bulk viscosity is written as (Heiselberg 1993; Lindblom et al. 1999; Lindblom \& Owen 2002; Nayyar \& Owen 2006)

$$
\frac{1}{\tau_{\mathrm{bv}}}=\frac{4 \pi}{690}\left(\frac{\Omega^{2}}{\pi G \bar{\rho}}\right)^{2} R^{2 l-2}\left[\int_{0}^{R} \rho r^{2 l+2} d r\right]^{-1} \int_{0}^{R} \zeta\left(\frac{r}{R}\right)^{6}\left[1+0.86\left(\frac{r}{R}\right)^{2}\right] r^{2} d r,
$$

where $R$ is the stellar radius and $\bar{\rho}=M /\left(4 \pi R^{3} / 3\right)$ is the averaged density of a non-rotating star (Madsen 1992). The bulk viscosity of unpaired SQM mainly depends on the rate of non-leptonic weak interaction: $\mathrm{u}+\mathrm{d} \leftarrow \mathrm{u}+\mathrm{s}$. In the high temperature limit $\left(T>10^{9} \mathrm{~K}\right)($ Madsen 2000$)$

$$
\zeta^{\text {high }} \approx 3.8 \times 10^{28} m_{100}^{4} \rho_{15}^{-1} T_{9}^{-2} \mathrm{~g} \mathrm{~cm}^{-1} \mathrm{~s}^{-1},
$$

the bulk viscosity in the low-temperature $\operatorname{limit}\left(T<10^{9} \mathrm{~K}\right)$ is (Madsen 2000)

$$
\zeta^{\text {low }} \approx 3.2 \times 10^{28} m_{100}^{4} \rho_{15} T_{9}^{2}(\kappa \Omega)^{-2} \mathrm{~g} \mathrm{~cm}^{-1} \mathrm{~s}^{-1},
$$

where $m_{100}$ is the strange quark mass $\left(m_{s}\right)$ in the unit of $100 \mathrm{MeV}$. For the dominant r-mode $(m=l=2)$, $\kappa=2 / 3$.

The dissipation timescale of shear viscosity is written as (Lindblom et al. 1998)

$$
\frac{1}{\tau_{\mathrm{sv}}}=(l-1)(2 l+1)\left[\int_{0}^{R} \rho r^{2 l+2} d r\right]^{-1} \int_{0}^{R} \eta r^{2 l} d r,
$$

The shear viscosity due to quark-quark scattering has been calculated for the case $T \ll \mu$ ( $T$ is the temperature and $\mu$ is the quark chemical potential) which can be presented as (Heiselberg 1993)

$$
\eta \approx 1.7 \times 10^{18}\left(\frac{0.1}{\alpha_{\mathrm{s}}}\right)^{5 / 3} \rho_{15}^{14 / 9} T_{9}^{-5 / 3} \mathrm{~g} \mathrm{~cm}^{-1} \mathrm{~s}^{-1}
$$


Due to pairing in the CFL phase, the shear viscosity induced by quark-quark scattering is suppressed by the factor $e^{\Delta / 3 k_{\mathrm{B}} T}$ and bulk viscosity is suppressed by the factor $e^{2 \Delta / k_{\mathrm{B}} T}$. Indeed, other viscosities are only dominant when these effects are exponentially suppressed in the CFL phase (Madsen 2000). For CSS, the viscosity at low temperature is determined by shear due to electron-electron scattering or by surface rubbing

$$
\tau_{\mathrm{sv}}^{\mathrm{ee}}=2.95 \times 10^{7}\left(\mu_{e} / \mu_{q}\right)^{-14 / 3} T_{9}^{5 / 3} \mathrm{~s},
$$

We take the maximized effect of electron shear $\mu_{\mathrm{e}} / \mu_{\mathrm{q}}=0.1$ (Madsen 2000). Surface rubbing due to the electron atmosphere being carried along by the r-modes in the quark phase, scattering mainly on photons in the nuclear crust, corresponds to a viscous time scale

$$
\tau_{\mathrm{sr}} \simeq\left(1.42 \times 10^{8}\right) s T_{9}(\nu / 1 \mathrm{kHz})^{-1 / 2},
$$

for a crust with maximal density (Madsen 2000).

By solving Equation (3) and different damping mechanisms numerically, we can derive the r-mode instability windows for USS and CSS. Fig. 1 shows the r-mode instability windows for USS with $M=$ $1.4 M_{\odot}$. The instability windows are calculated with different parameters of EOS, which are constrained by two-solar-mass pulsar observations and tidal deformability of the merging binary. Also indicated are the spin frequency and core temperature of compact stars in LMXBs, which have been given in Gusakov et al. 2014, and temperature for HETE J1900.1-2455 $\left(5 \times 10^{5} \mathrm{~K}\right)$ was updated in Degenaar et al. 2017. The left panel is for $a_{4}=0.61,0.72$ and 0.83 , and $B_{\text {eff }}^{1 / 4}=138 \mathrm{MeV}$. The right one is for $B_{\text {eff }}^{1 / 4}=136 \mathrm{MeV}, 138 \mathrm{MeV}$ and $140 \mathrm{MeV}$, and $a_{4}=0.72$. We find that $a_{4}$ and $B_{\text {eff }}^{1 / 4}$ do not have significant effects on the r-mode instability window at high temperature (approximately higher than $10^{7} \mathrm{~K}$ ); r-mode instability windows at low temperature are shrinking while $a_{4}$ or $B_{\text {eff }}^{1 / 4}$ are increasing. A smaller allowed $a_{4}$ will lead to a larger r-mode instability window.

Since some pulsar like objects might be low mass strange stars (Zhou et al. 2017), we present the r-mode instability windows for USS with different masses in Fig.2, especially for mass $<1.0 M_{\odot}$. The windows become larger while the star mass increases. Many sources are around right boundaries of instability windows and SAX J1808.4-3658 is in the instability region even though the star mass is $0.5 M_{\odot}$. The sources are out of the instability region when the mass is $0.1 M_{\odot}$.

The r-mode instability windows for CSS with different gaps and effective bag constants are displayed in Fig. 3 and Fig.4. The spin frequency and core temperature of young pulsars are listed in Table1, and their core temperature has been inferred by assuming an outer envelope completely composed of a crust of iron elements (Becker 2009). We consider the shear due to surface rubbing and electron-electron scattering respectively in Fig. 3 and 4. It is shown that the suppressed effect of surface rubbing for the instability window is much stronger than that for shear by electron-electron scattering. In addition, the viscous timescale for shear due to surface rubbing is $\tau_{s r} \propto\left(10^{8}-10^{10}\right) T_{9}$ and the viscous timescale for shear due to electronelectron scattering is $\tau_{\mathrm{sv}}^{\mathrm{ee}} \propto 10^{12} \mathrm{~T}_{9}^{5 / 3}$. This implies that the dominant role in suppressing the r-modes is supposed to be the shear due to surface rubbing. In order to show the damping effect from the existence of a crust, we consider the shear due to either surface rubbing or electron-electron scattering in the following 

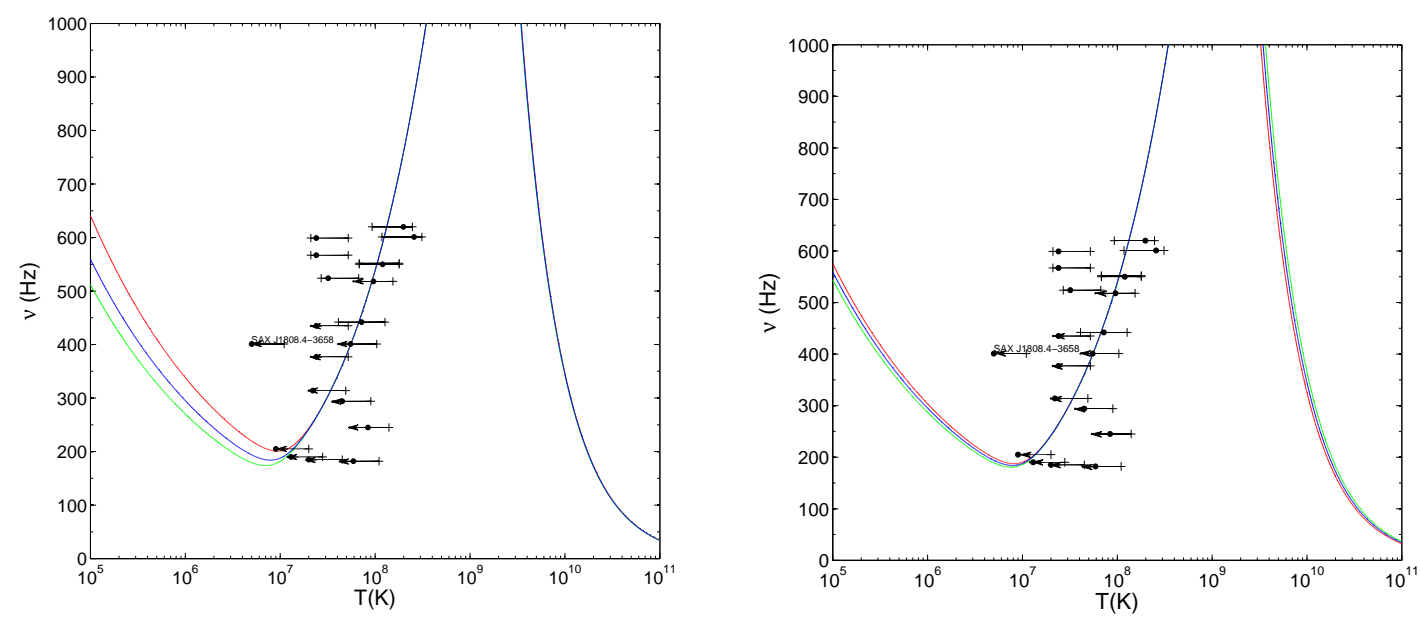

Fig. 1: R-mode instability windows for USS with $M=1.4 M_{\odot}$. The observed spin frequency and core temperature of LXMBs are also included for a comparison. The left panel is for different $a_{4}$ with $B_{\mathrm{eff}}^{1 / 4}=$ $138 \mathrm{MeV}$ and the right one is for different $B_{\text {eff }}^{1 / 4}$ with $a_{4}=0.72$. In the left panel, the green line, blue line and red line correspond to $a_{4}=0.61,0.72$ and 0.83 respectively. In the right panel, the green line, blue line and red line signify $B_{\text {eff }}^{1 / 4}=136,138$ and $140 \mathrm{MeV}$ respectively.

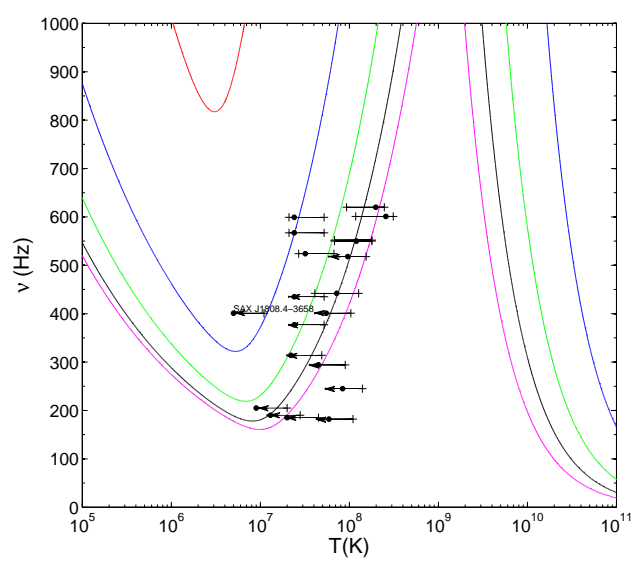

Fig. 2: Similar to Fig.1,but for USS with $M=0.1 M_{\odot}($ red line $), M=0.5 M_{\odot}($ blue line $), M=$ $1.0 M_{\odot}($ green lien $), M=1.5 M_{\odot}$ (black line $)$ and $M=2.0 M_{\odot}($ mauve line $)$. The corresponding parameters for the EOSs are set as $B_{\text {eff }}^{1 / 4}=138 \mathrm{MeV}$ and $a_{4}=0.72$.

In Fig. 3 and 4, most of the young pulsars are located outside the instability region, except for the fast spinning PSR J0537-6910 and the Crab. Different gaps as well as different effective bag constants and $a_{4}$ lead to a slight influence on the r-mode instability windows of CSS.The windows at high temperature dramatically shrink when the gaps decrease to $\Delta=1 \mathrm{MeV}$, which are shown in the right panel in Fig.4. No matter if shear due to surface rubbing or electron-electron scattering is considered, PSR J0537-6910 is always in the instability region, but when a star damped by shear due to surface rubbing is examined, Crab is out of the instability windows.

In Fig.5, the r-mode instability windows for CSS with different $\operatorname{star} \operatorname{masses}(M=$ 

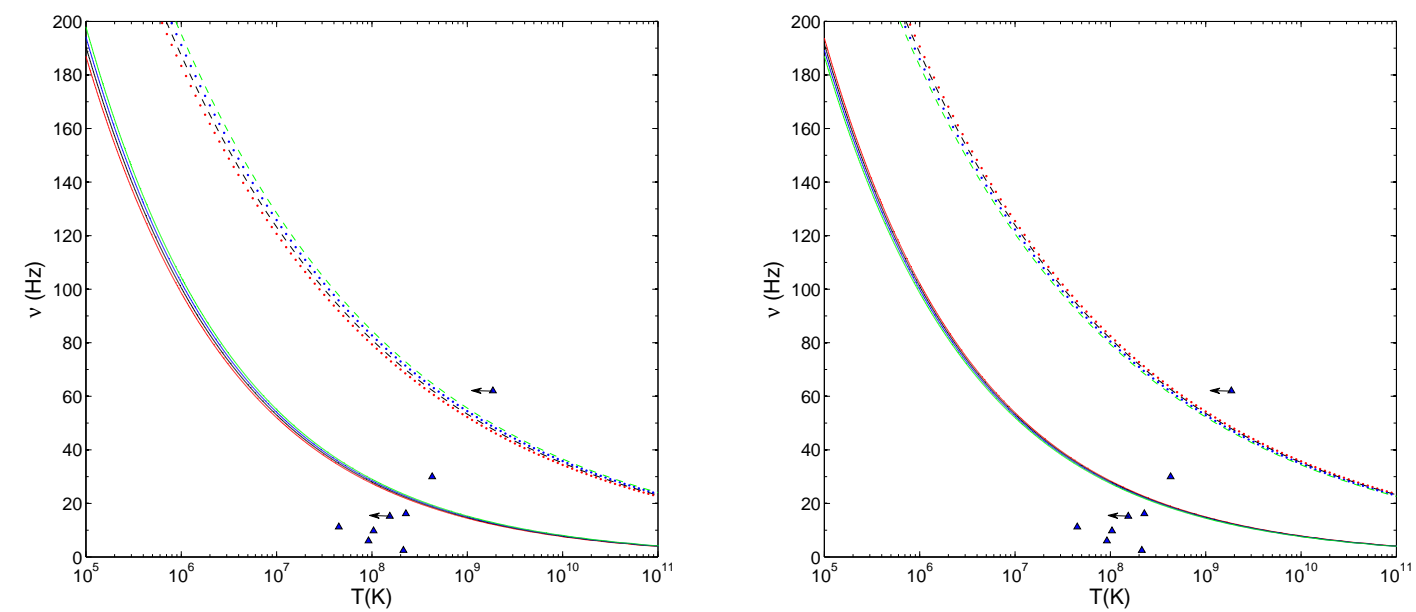

Fig. 3: R-mode instability windows for CSS with $a_{4}=1$ and $M=1.4 M_{\odot}$. The observed spin frequency and core temperature of young pulsars tabulated in Table 1 are also included for comparison(blue triangles). The left panel is for different $B_{\text {eff }}^{1 / 4}$ with $\Delta=100 \mathrm{MeV}$, and the red, black, blue and green lines correspond to $B_{\text {eff }}^{1 / 4}=146,148.5,151,153.5 \mathrm{MeV}$ respectively. The right panel is for different $\Delta$ with $B_{\text {eff }}^{1 / 4}=146 \mathrm{MeV}$, and the red, black, blue and green lines represent $\Delta=70,80,90,100 \mathrm{MeV}$ respectively. Dotted curves or dashed curves mean shear due to surface rubbing, and solid curves signify shear due to electron-electron scattering

Table 1: Spin frequency and surface temperature for young pulsars that have measurements (or upper limits) of both. Spin frequency for young pulsars are taken from the ATNF catalog (Manchester et al. 2005).

\begin{tabular}{lrrrr}
\hline Source & $\begin{array}{c}T_{s}^{\infty} \\
(\mathrm{Hz})\end{array}$ & $\begin{array}{c}T_{\text {core }}(\mathrm{Fe}) \\
\left(10^{6} \mathrm{~K}\right)\end{array}$ & $\left(10^{8} \mathrm{~K}\right)$ & References \\
\hline PSR B0531+21(Crab) & 29.95 & 1.79 & 4.29 & Weisskopf et al. 2004 \\
PSR J0205+6449 & 15.22 & $<1.02$ & 1.54 & Slane et al. 2004 \\
PSR J1119-6127 & 2.451 & 1.22 & 2.14 & Safi-Harb \& Kumar 2008 \\
PSR J1357-6429 & 6.020 & 0.77 & 0.92 & Zavlin 2007 \\
PSR B0833-45(Vela) & 11.20 & 0.52 & 0.45 & Manzali et al. 2007 \\
PSR B1706-44 & 9.760 & 0.82 & 1.04 & McGowan et al. 2004 \\
PSR J0537-6910 & 62.03 & $<4.00$ & 18.58 & Andersson et al. 2018 \\
PSR J1833-1034 & 16.16 & 1.26 & 2.27 & Matheson \& Safi-Harb 2010 \\
\hline
\end{tabular}

crease with the star mass gradually increasing. With small mass $\left(<1.0 M_{\odot}\right)$, the two fast spinning pulsars might be outside of these windows.

\section{CONCLUSIONS AND DISCUSSION}

Coupled with a new constraint on EOS, we investigate the r-mode instability windows of USS and CSS. Moreover, these r-mode instability windows are compared with the spin frequency and core temperature of compact star in LMXBs/millisecond pulsars (MSPs) and young pulsars. The effects of EOS for unpaired SQM are only dominant at low temperature $\left(T \lesssim 10^{7} \mathrm{~K}\right.$ ). For CSS, the damping mechanisms due to the 

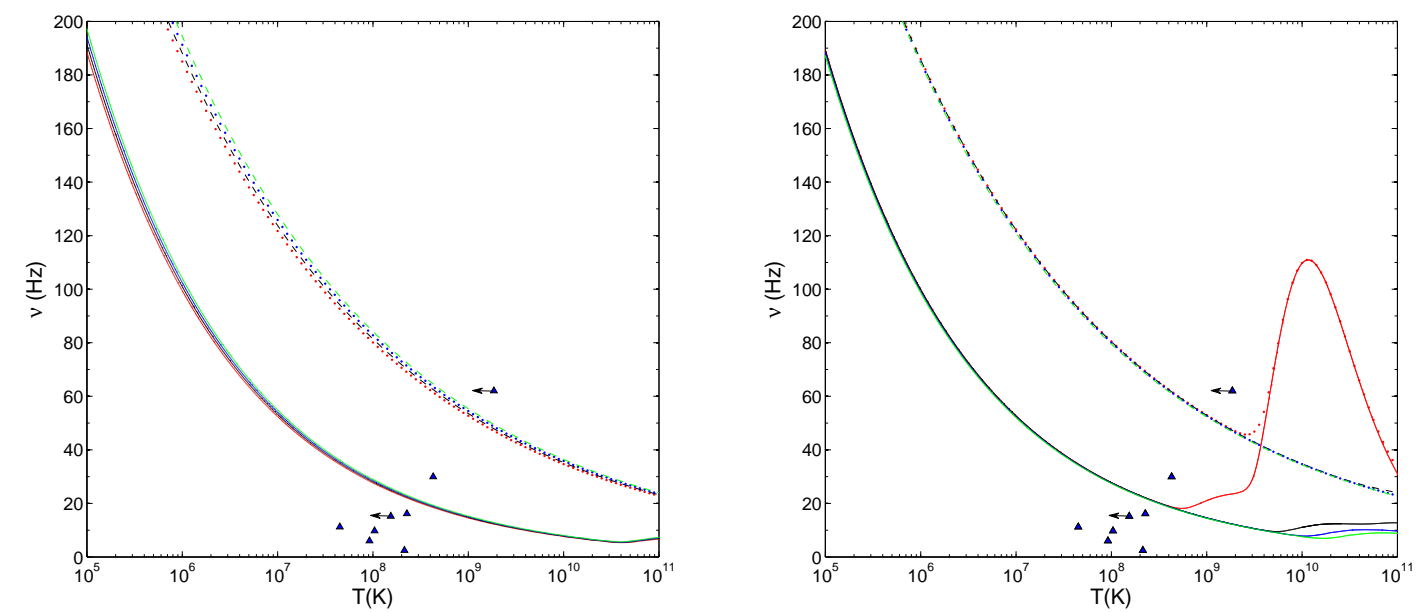

Fig. 4: R-mode instability windows for CSS with $a_{4}=0.61$ and $M=1.4 M_{\odot}$. The observed spin frequency and core temperature of young pulsars tabulated in Table 1 are also included for comparison(blue triangles). Dotted curves or dashed curves correspond to shear due to surface rubbing and solid curves indicate shear due to electron-electron scattering. The left panel is for different $B_{\text {eff }}^{1 / 4}$ with $\Delta=75 \mathrm{MeV}$, and the red , black, blue and green lines represent $B_{\mathrm{eff}}^{1 / 4}=144,146,148,150 \mathrm{MeV}$ respectively. The right panel is for different gaps with $B_{\mathrm{eff}}^{1 / 4}=136.5 \mathrm{MeV}$, and the red, black, blue and green lines correspond to $\Delta=$ $1,10,20,30 \mathrm{MeV}$ respectively.

R-mode instability has been identified as a viable and promising target for gravitational wave searches and has been taken into account in recent analysis. LMXBs and young pulsars are possible sources for detecting gravitational waves driven by r-mode (Kokkotas \& Schwenzer 2016). USS seems consistent with LMXB data except for SAX J1808.4-3658. This source is located in the instability region unless the mass is smaller than $0.5 M_{\odot}$ and therefore could experience r-mode-driven spin-down. However, the measured spindown rate is consistent with that caused by a canonical LMXB magnetic field $\left(\sim 10^{8} \mathrm{G}\right)$, hence suggesting that the r-mode instability is not the dominant effect and SAX J1808.4-3658 may be a massive star (Salmi et al. 2018). This apparent tension between the minimum damping r-mode model of USS and the spintemperature-mass data of SAX J1808.4-3658 may be in conflict with each other. That means additional damping mechanisms might work in this system, which could modify the instability window.

The shear viscosity due to quark scattering and bulk viscosity are suppressed in the CFL phase, which lead to disagreement with the observational data of LMXBs (Alford et al. 2008). Actually, a CSS may have a tiny nuclear crust (Madsen 2000). As shown in this work, EOSs do not have a significant effect on the instability windows of CSS. The most effective dissipation mechanisms are the shear due to surface rubbing or electron-electron scattering. PSR J0537-6910 is always located in the instability window no matter if shear due to surface rubbing or electron-electron scattering is considered. From this point of view, the fast spinning young pulsar PSR J0537-6910 is extremely promising source for detecting gravitational waves from a rotating strange star. In addition to this, we could learn something about the structure and mass of PSR J0537-6910 or Crab by comparing the r-mode instability with observational data.

Moreover, a radio observation recently demonstrated that PSR J0537-6910 appears to be close to the 

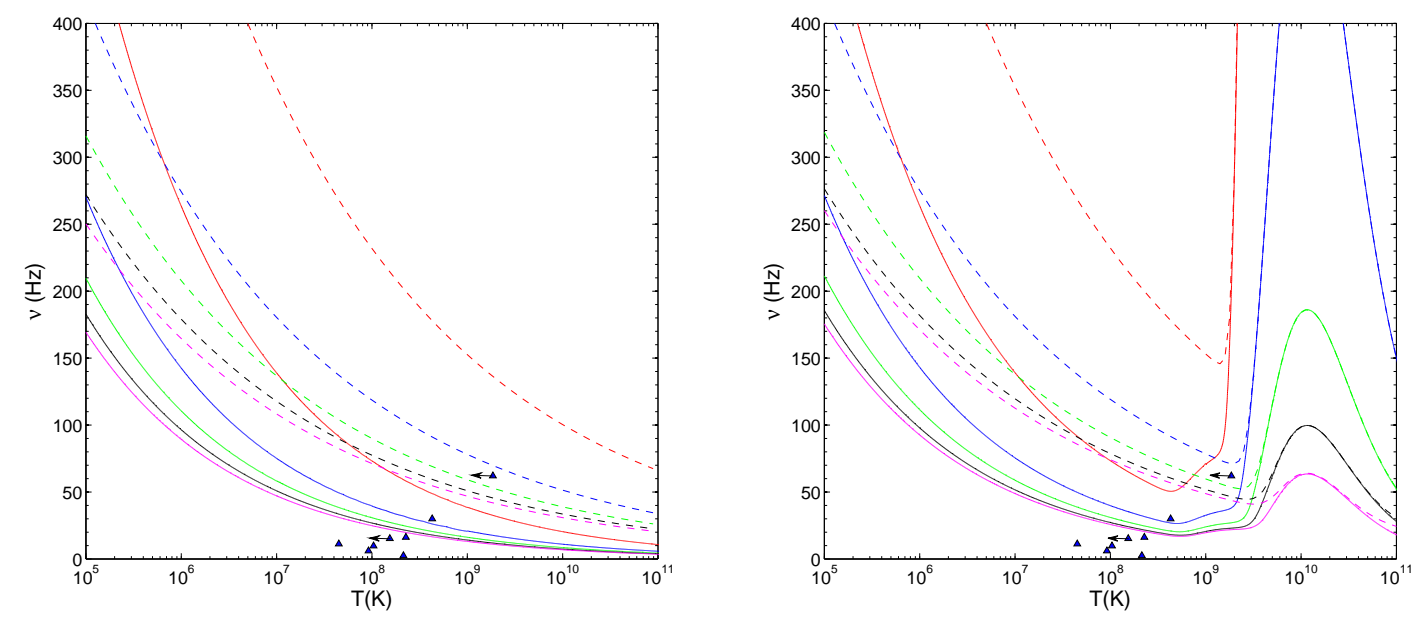

Fig. 5: R-mode instability windows for CSS with different masses. The observed spin frequency and core temperature of young pulsars tabulated in Table 1 are also included for comparison(blue triangles). The left panel is for $a_{4}=1, \Delta=100 \mathrm{MeV}$ and $B_{\text {eff }}^{1 / 4}=146 \mathrm{MeV}$. The right panel is for $a_{4}=0.61, \Delta=1 \mathrm{MeV}$ and $B_{\mathrm{eff}}^{1 / 4}=136.5 \mathrm{MeV}$. The red, blue, green, black and mauve lines correspond to $M=0.1 M_{\odot}, M=$ $0.5 M_{\odot}, M=1.0 M_{\odot}, M=1.5 M_{\odot}, M=2.0 M_{\odot}$ respectively. Dashed (solid) curves signify shear due to surface rubbing (electron-electron scattering).

radiation can possibly be detected by advanced LIGO(aLIGO) (Andersson et al. 2018). The results above suggest that the case for USS has difficulty in agreeing with the radio observation (Andersson et al. 2018). In addition, if the mass of PSR $\mathbf{J} 2215+5135$ is confirmed $\left(M=2.27_{-0.15}^{+0.17} M_{\odot}\right)$, USS would be rule out since the tidal deformability measurement of GW170817 implies $M \leq 2.18 M_{\odot}$ (Linares et al. 2018; Zhou et al. 2018). If these case turn out to be ture, young pulsars might be CFL phase strange stars.

Acknowledgements We thank the referee for valuable suggestions that have allowed us to improve our manuscript significantly. The work was supported by the National Natural Science Foundation of China Nos. 11373006, 11873040, U1838108, U1531137.

\section{References}

Aaij, R., Adeva, B., Adinolfi, M., et al. 2016, Chinese Physics C, 40, 0110013

Abbott, B. P., Abbott, R., Abbott, T. D., et al. 2017, Physical Review Letters, 119, 1611012

Alford, M., Rajagopal, K., Reddy, S., \& Wilczek, F. 2001, Phys. Rev. D, 64, 0740173

Alford, M. G., Schmitt, A., Rajagopal, K., \& Schäfer, T. 2008, Reviews of Modern Physics, 80, 1455 2, 3 , 8

Alford, M. G., \& Schwenzer, K. 2014a, ApJ, 781, 261

Alford, M. G., \& Schwenzer, K. 2014b, Physical Review Letters, 113, 2511022

Andersson, N. 1998, ApJ, 502, 7081

Andersson, N., Kokkotas, K. D., \& Stergioulas, N. 1999, ApJ, 516, 3071

Andersson, N., Jones, D. I., \& Kokkotas, K. D. 2002, MNRAS, 337, 1224 1, 2 
Becker W., 2009, Neutron Stars and Pulsars. (Berlin: Springer) 2, 5

Bhattacharyya, S., Bombaci, I., Logoteta, D., \& Thampan, A. V. 2016, MNRAS, 457, 31013

Chandrasekhar, S. 1970, Physical Review Letters, 24, 6111

Chugunov, A. I., Gusakov, M. E., \& Kantor, E. M. 2017, MNRAS, 468, 2912

Degenaar, N., Ootes, L. S., Reynolds, M. T., Wijnands, R., \& Page, D. 2017, MNRAS, 465, L10 5

Fraga, E. S., Pisarski, R. D., \& Schaffner-Bielich, J. 2001, Phys. Rev. D, 63, 1217023

Friedman, J. L., \& Schutz, B. F. 1978, ApJ, 222, 2811

Friedman, J. L., \& Morsink, S. M. 1998, ApJ, 502, 7141

Glendenning, N. K., \& Weber, F. 1992, ApJ, 400, 6472

Gusakov, M. E., Chugunov, A. I., \& Kantor, E. M. 2014, Phys. Rev. D, 90, 063001 2, 5

Haskell, B., Degenaar, N., \& Ho, W. C. G. 2012, MNRAS, 424, 932

Heiselberg, H., \& Pethick, C. J. 1993, Phys. Rev. D, 48, 29164

Hessels, J. W. T., Ransom, S. M., Stairs, I. H., et al. 2006, Science, 311, 1901

Kantor, E. M., Gusakov, M. E., \& Chugunov, A. I. 2016, MNRAS, 455, 7392

Kokkotas, K. D., \& Schwenzer, K. 2016, European Physical Journal A, 52, 38 2, 8

Li, A., Zhu, Z.-Y., \& Zhou, X. 2017, ApJ, 844, 413

Linares, M., Shahbaz, T., \& Casares, J. 2018, ApJ, 859, 549

Lindblom, L., Owen, B. J., \& Morsink, S. M. 1998, Physical Review Letters, 80, 4843 1, 2,4

Lindblom, L., Mendell, G., \& Owen, B. J. 1999, Phys. Rev. D, 60, 0640064

Lindblom, L., \& Owen, B. J. 2002, Phys. Rev. D, 65, 0630064

Madsen, J. 1992, Phys. Rev. D, 46, 32904

Madsen, J. 1998, Physical Review Letters, 81, 33111

Madsen, J. 2000, Physical Review Letters, 85, 10 2, 4, 5, 8

Mahmoodifar, S., \& Strohmayer, T. 2013, ApJ, 773, 140 1, 2

Manchester, R. N., Hobbs, G. B., Teoh, A., \& Hobbs, M. 2005, AJ, 129, 19937

Manzali, A., De Luca, A., \& Caraveo, P. A. 2007, ApJ, 669, 5707

Matheson, H., \& Safi-Harb, S. 2010, ApJ, 724, 5727

McGowan, K. E., Zane, S., Cropper, M., et al. 2004, ApJ, 600, 3437

Nayyar, M., \& Owen, B. J. 2006, Phys. Rev. D, 73, 0840014

Owen, B. J., Lindblom, L., Cutler, C., et al. 1998, Phys. Rev. D, 58, 084020 1, 4

Pi, C.-M., Yang, S.-H., \& Zheng, X.-P. 2015, RAA, 15, 8712

Rajagopal, K., \& Wilczek, F. 2001, Physical Review Letters, 86, 34923

Safi-Harb, S., \& Kumar, H. S. 2008, ApJ, 684, 5327

Salmi, T., Nättilä, J., \& Poutanen, J. 2018, preprint (arXiv:1805.01149) 8

Slane, P., Helfand, D. J., van der Swaluw, E., \& Murray, S. S. 2004, ApJ, 616, 4037

Wang, J.-S., \& Dai, Z.-G. 2017, A\&A, 603, A9 1

Weissenborn, S., Sagert, I., Pagliara, G., Hempel, M., \& Schaffner-Bielich, J. 2011, ApJ, 740, L14 3

Weisskopf, M. C., O’Dell, S. L., Paerels, F., et al. 2004, ApJ, 601, 10507

Zavlin, V. E. 2007, ApJ, 665, L143 7 
Zdunik, J. L. 2002, A\&A, 394, 6412

Zhou, X., Tong, H., Zhu, C., \& Wang, N. 2017, MNRAS, 472, 24035

Zhou, E.-P., Zhou, X., \& Li, A. 2018, Phys. Rev. D, 97, 083015 2, 3, 9 